\title{
Scrub Typhus Meningoencephalitis
}

\author{
Masaraf Hussain \\ Additional information is available at the end of the chapter \\ http://dx.doi.org/10.5772/intechopen.68369
}

\begin{abstract}
Scrub typhus is a zoonotic disease, which is characterized by an acute febrile illness, lymphadenopathy, eschar, multisystem involvement, and a rapid response to doxycycline. It is found widely in Asia and Pacific islands. Various neurological manifestations, like meningoencephalitis, cranial nerve involvement, are known to occur. Diagnosis is based on a high clinical suspicion, presence of eschar and serological tests. Prompt diagnosis and management can prevent morbidity and mortality, as it has a rapid response to doxycycline. However, due to underdiagnosis, it has often been called as the 'forgotten killer'.
\end{abstract}

Keywords: scrub typhus, neurological manifestation, meningitis, encephalitis, meningoencephalitis

\section{Introduction}

Scrub typhus is also known as 'tsutsugamushi disease'. It is a zoonotic disease. This disease was first mentioned in Japanese folklore to be associated with the jungle mite or 'chigger', which was named 'dangerous bug'. Therefore, the term 'tsutsugamushi' is derived from two Japanese words, 'tsutsuga' which means something small and dangerous, and 'mushi' which means creature.

'Typhus' has been derived from the Greek word 'typos' which means 'fever with stupor'. The name itself reveals a clinical aspect of the disease [1].

\section{Scrub typhus}

\subsection{Epidemiology}

Although scrub typhus was known in China in the third century A.D., it was first described by Hakuju Hashimoto in 1810 in people living in the banks of the Shinano river and later 
in 1879 by Baclz and Kawakami as Japanese 'flood fever'. It is widespread in the so-called 'tsutsugamushi triangle' which extends from Pakistan, India, Nepal in the West, to South Eastern Siberia, Japan, China and Korea in the North, to Indonesia, the Philippines, Northern Australia and the Pacific islands in the South. Taiwan is the centre of the tsutsugamushi triangle, and Korea has the highest reported incidence in the world [2]. About one million new cases are identified annually.

In India, the disease had occurred among troops during World War II in the state of Assam and West Bengal. Although the disease is endemic in India, epidemics have also been reported.

Epidemiological reports confirm strong existence of scrub in hilly/rain prone areas. Outbreaks reveal an autumn-winter type and a summer type of pattern. In comparison with the summer type, the autumn-winter type is less severe.

Increasing prevalence of scrub typhus reported from some Asian countries may be related to urbanization of rural areas.

\subsection{Pathophysiology}

Scrub typhus is caused by an obligate intracellular gram-negative bacterium called Orientia tsutsugamushi. Ogata in 1931 isolated the organism and named it Rickettsia tsutsugamushi. Now it has been renamed as O. tsutsugamushi. The organism lacks a cell wall. There are six important serotypes of O. tsutsugamushi-Gilliam, Karp, Kato, Shimokoshi, Kawasaki and Kuroki. A new strain has been isolated from a case of scrub typhus in Australia which was quite different from the classic strains and has been named as Litchfield.

The mite has four stages: egg, larva, nymph and adult. The larval forms (chiggers) transmit the disease to humans and other vertebrates. The larvae feed on rodents particularly wild rats of subgenus Rattus, The infection in humans is acquired during outdoor recreational and agricultural activities, by the bite of the larval stage of mites. The areas are usually secondary scrub growth which grows after clearance of primary forest hence the term scrub typhus. Humans are therefore accidental hosts for the pathogen. Vertical transovarial transmission occurs in mites. One case of transplacental spread has been reported in a pregnant woman, who delivered a preterm baby with scrub typhus IgM positivity.

Following the bite, the pathogen multiplies at the site of inoculation and produces both systemic and local manifestation. Infection spreads through both haematogenous and lymphatic routes. The severity of the illness depends on both host-related and pathogen-related factors. Pathogenrelated factors may be related to the different strains of $O$. tsutsugamushi. Host-related factors as seen in humans with G6PD deficiency who has a worse prognosis also play a role. Target cells for multiplication are the endothelial cells of the various systems. The immune response induced by the pathogen is a combination of humoral and cell-mediated immunity. There occurs a rise of cytokines during an acute infection. There also occurs a rise in macrophage colony stimulating factor, interferon gamma and granulocyte colony stimulating factor. Therefore, the macrophage and T-lymphocyte response may be the main factor in immunity against the infection. However, the parasite has also evolved to evade the immune response of the host. The pathogen can down-regulate the expression of glycoprotein 96, in infected macrophages and endothelial 
cells and thereby neutralize host immune response. This molecule plays a central role antigen presentation and antibody production [3].

Central nervous system involvement occurs in scrub typhus. It is speculated that since the pathogen is an obligatory intracellular organism, it enters the cerebrospinal fluid in a monocyte or grow through the endothelium, enter via the luminal cell membrane and release into the perivascular space [4]. The pathogen also invades and multiplies in the vascular endothelium and cause wide spread vasculitis. Autopsy specimens have shown central nervous system pathology in scrub typhus cases in the form of diffuse or focal mononuclear cell infiltration of the leptomeninges, presence of typhus nodules (clusters of microglial cells) and haemorrhages of the brain substance [4].

\subsection{Clinical features}

The incubation period of $O$. tsutsugamushi in humans is around 10-12 days (can vary between 6 and 21 days). The clinical manifestations vary from a mild febrile illness to a severe potentially disease. The systemic features of the infection include fever, gastrointestinal disturbance, malaise, cough, myalgia and headache. A maculopapular rash starting from the trunk and spreading to the limbs is seen towards the end of the first week of the fever. Diffuse lymphadenopathy is commonly observed.

A necrotic 'eschar' at the bite site is almost diagnostic of scrub typhus (Figure 1).

The eschar resembles skin burn of cigarette butt. Eschar is found in 7-80\% patients of scrub typhus [3]. In the authors study, eschar was detected in $28.81 \%$ of the patients of scrub typhus and $30.77 \%$ patients of meningoencephalitis due to scrub typhus [6]. The wide range of detection may be due to the difficulty in detecting eschars in dark-skinned individuals, difference in the eschar inducing capacity of the different strains of $O$. tsutsugamushi. The groin, axilla, waist and other exposed parts of the body are common sites of eschar detection. In the authors

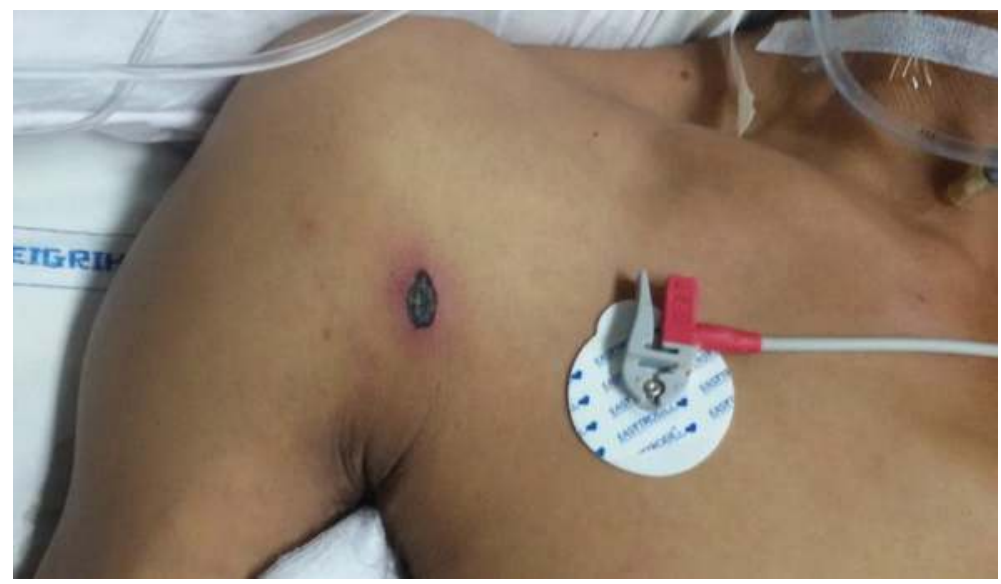

Figure 1. Eschar at bite site. 
study, eschar was mostly found in the inguinal region. Different pattern of eschar distribution found in males and females due to the differences in skin folds, clothing and pressure points created by garments. The eschar not only has immense diagnostic relevance but is also important prognostically. Absence of an eschar is a risk factor for mortality [2].

Neurological involvement is often a prominent clinical manifestation of scrub typhus. However, they are still an unclear entity. Meningitis or meningoencephalitis can occur in upto one-fifth of affected patients. In the authors study, meningoencephalitis was found in $13.2 \%$ of scrub typhus patients [6]. The various neurological manifestations of scrub typhus [5] are as given in (Table 1).

Meningitis has features of headache, vomiting, fever, neck stiffness, along with cerebrospinal fluid (CSF) pleocytosis. Altered sensorium and fever with CSF pleocytosis are features of encephalitis. Altered sensorium with fever but normal CSF is found in encephalopathy.

Neurological manifestations in scrub typhus does not occur in isolation but are accompanied by systemic features like jaundice, breathlessness, cough, renal impairment and in some cases, with multi-organ dysfunction. In the authors study [6] neurological manifestations were associated with lymphadenopathy $(46.15 \%)$, jaundice $(53.85 \%)$, pulmonary oedema $(23.08 \%)$, oliguria $(15.38 \%)$, hepatomegaly (38.46\%) and splenomegaly (7.69\%). Multi-organ dysfunction was found in $15.38 \%$ patients of scrub typhus with neurological manifestation.

The most common symptom of scrub typhus is fever. The fever is usually mild and accompanied by myalgia. In the authors study the mean duration of fever was 5.61 days, prior to meningoencephalitis presentation.

Headache is a common symptom in scrub typhus (46-77\%). A severe holocranial headache almost invariably occurs and thereby helps in identifying suspected cases. Headache occurs

Direct involvement

1. Meningitis

2. Meningoencephalitis

3. Encephalitis

4. Encephalopathy

5. Seizure

6. Stroke

Immune mediated

1. Optic neuritis

2. Myelitis

3. Acute-disseminated encephalomyelitis

4. Neuropathy

Table 1. Neurological involvement in scrub typhus. 
not only in patients of meningitis or meningoencephalitis but in other scrub typhus patients also. However, in those cases, the headache is less severe.

In scrub typhus meningitis, the severe headache is associated with neck stiffness and fever. Other signs of meningeal irritation like Kernig's sign may also be present. These meningeal signs are detected in upto $45 \%$ patients. In the author's study, meningeal signs were present in $76.92 \%$ patients [6].

Altered sensorium is present in scrub typhus patient with encephalitis and meningoencephalitis. In the author's study, altered sensorium was found in all patients; however, other studies have reported a lower incidence.

Seizure occurs in scrub typhus with neurological involvement in $22-50 \%$ cases, though uncommon myoclonic seizure was found in one patient in the author's series.

Cranial nerve deficits are seen in $25 \%$ patients. Most commonly sixth nerve involvement is seen, which maybe unilateral or bilateral. Facial palsy may occur in isolation or in association with Guillain Barre syndrome [2]. Cochlear nerve involvement occurs in about 19\% patients and cause sensorineural hearing loss, otalgia and tinnitus. This may be due to direct invasion by the pathogen or due to a secondary immune-mediated effect.

Other uncommon neurological manifestations of scrub typhus mentioned are infarction, cerebellitis, haemorrhages, subdural hematoma and Guillain Barre syndrome [2].

\subsection{Diagnosis of scrub typhus}

This is aided by serological tests in appropriate clinical setting.

Microimmunofluorescence is considered the test of choice. However, lack of fluorescent microscopes makes it difficult for most hospitals.

Latex agglutination, indirect haemagglutination, immunoperoxidase assay, ELISA and polymerase chain reaction (PCR) are also available. The nested PCR is more sensitive than the serological tests.

PCR can be used to detect rickettsial DNA in both blood and eschar samples. The PCR is targeted at the gene encoding the major 56-kDa antigen and/or 47-kDa surface antigen gene. The results are best within first week for blood samples.

ELISA (IgG and IgM) technique, particularly immunoglobulin M (IgM), capture assays are probably the most sensitive test available for rickettsial diagnosis. In cases of infection with O. tsutsugamushi, a significant IgM antibody titre is observed at the end of the first week, whereas IgG antibodies appear at the end of the second week.

Weil Felix test: the sharing of the antigen between rickettsia and proteus is the basis of this heterophile antibody test. Though this test lacks high sensitivity and specificity, it is inexpensive. The test should be carried out after 5-7 days of onset of fever.

However, due to the antigenic diversity of the pathogen, a battery of tests may be required for the diagnosis [1]. 
CSF analysis in scrub typhus meningoencephalitis reveals mild-to-moderate elevation in protein, low-to-normal glucose and mild degree of lymphocytic pleocytosis. By using nested PCR, the genotypes invading the central nervous system (CNS) may be identified. By this, it was suggested that the Karp and Boryong genotypes possibly invade the CNS more than other types [4]. In the author's study, tuberculous meningitis remained the close differential diagnosis of scrub typhus meningitis due to similar CSF findings [6]. However, CSF adenosine deaminase (ADA) may be helpful, as it is elevated $>10$ in tuberculous meningitis, unlike scrub typhus meningitis.

Neurological involvement in scrub typhus is usually associated with a normal MRI and nonspecific EEG slowing. Often MRI of brain in scrub typhus may reveal features of ischemic changes due to vasculitis or parainfectious demyelination [5].

\subsection{Treatment}

Doxycycline is the drug of choice. It is bacteriostatic to O. tsutsugamushi but does not cross the blood brain barrier beyond 15-30\% [2]. Therefore, in some instances, neurological deterioration can continue despite doxycycline therapy. This may be due to resistance, immune-mediated injury, or due to drug interactions with oral antacids. Doxycycline is given in the dose of $200 \mathrm{mg} /$ day, in two divided doses, for individuals above $45 \mathrm{kgs}$ body weight, for a duration of 7 days. In children, doxycycline is given in the dose of $4.5 \mathrm{mg} / \mathrm{kg}$ body weight in two divided doses. Doxycycline is contraindicated in pregnant women. In complicated cases, intravenous is given followed by oral doxycycline to complete 7-15 days of therapy.

Azithromycin is another drug which can be used in a dose of $500 \mathrm{mg}$ daily for 5 days and $10 \mathrm{mg} / \mathrm{kg}$ body weight in children for 5 days. It can also be given intravenously in complicated cases. Azithromycin is the drug of choice in pregnant women with scrub typhus. It is also preferred in patients of scrub typhus with renal failure, where doxycycline is not given.

In complicated cases, Chloramphenicol can also be used. It is administered intravenously at a dose of $50-100 \mathrm{mg} / \mathrm{kg} /$ day, 6 hourly doses, followed by oral therapy to complete $7-15$ days of therapy.

Doxycycline and/or chloramphenicol resistant strains have been detected in South-East Asia. These strains are sensitive to Azithromycin.

Patients with meningoencephalitis due to scrub typhus can be additionally administered with dexamethasone, or mannitol, if they have altered sensorium or cranial nerve deficits.

Recovery is usually brisk with appropriate therapy.

Pre-antibiotic era mortality was more than $60 \%$; however, recent data show a mortality of approximately $30 \%$ [2]. In the author's study [6], the mortality was $15.38 \%$. Mortality is usually associated with multi-organ dysfunction syndrome.

\section{Conclusion}

Neurological complication is not uncommon in scrub typhus. They present with acute febrile illness with altered sensorium and meningeal signs. The presence of 'eschar' helps in early 
diagnosis, but they are often absent. Prompt CSF analysis is required on clinical suspicion of neurological features in scrub typhus patients. Timely initiation of therapy results in recovery and less complications.

\section{Author details}

Masaraf Hussain

Address all correspondence to: masarafhussain@yahoo.co.in

Department of Neurology, North Eastern Indira Gandhi Regional Institute of Health and Medical Sciences, Shillong, India

\section{References}

[1] Mahajan SK. Scrub typhus. JAPI. Nov 2005;Vol. 53:954-957

[2] Vishwanath S, Muthu V, Iqbal N, Bhavith R. Scrub typhus meningitis in South India-A retrospective study. PLOS ONE. /www.plosone.org June 2013/Volume 8/Issue 6/e66595

[3] Rajapakse S, Rodrigo C, Fernando D. Asian Pacific Journal of Tropical Medicine. April 2012;Vol. 5(Issue 4):253-57

[4] Pai H, Sohn S, Scong Y. Central nervous system involvement in patients with scrub typhus. Clinical Infectious Diseases. 1997;24:436-440

[5] Jamil MD, Hussain M, Lyngdoh M, Sharma S, Barman B. Scrub typhus meningoencephalitis, a diagnostic challenge for clinicians: A hospital based study from North East India. Journal of Neuroscience in Rural Practice. 2015;Vol. 6(Issue 4):488-493. DOI: 10.4103/0976-3147.169769

[6] Misra UK, Kalita J, Mani VE. Neurological manifestations of scrub typhus. Journal of Neurology, Neurosurgery, and Psychiatry. 2015;86:761-766 
